\title{
Acquisition, discrimination, and extinction with intracranial stimulation'
}

GARY DEATHERAGE?

Two brain areas, the ventromedial nucleus of the hypothalamus and the septum, were compared on each of three variables for intracranial electrical stimulation: acquisition, discrimination, and extinction. The ventromedial nucleus groups had significantly higher response rates during acquisition, significantly more responses during the correct interval in discrimination, and made more responses to extinction than the septal implant groups. Nondiscrimination controls revealed that discrimination training had no effect upon resistance to extinction for either brain area group.

Olds (1956), Olds (1960), and Olds \& Olds (1963) have demonstrated that rate of response for intracranial stimulation is a function of the brain area stimulated. It has not been demonstrated, however, that extinction or proficiency at discrimination is a function of brain area stimulated. It was the purpose of this preliminary study to investigate such a possibility.

\section{Method}

Sixteen $280-320 \mathrm{gm}$ male albino rats were the Ss. Seven Ss were stereotaxically implanted in the septum with stainless steel bipolar electrodes, and seven Ss were similarly implanted in the ventromedial nucleus of the hypothalamus. Two Ss served as nonimplanted normal controls.

There were three phases to the experiment:

Phase I. Following a three day recovery from surgery all 16 Ss were given training for four daily sessions of $30 \mathrm{~min}$. each in a modified Skinner box. Each bar press delivered a 60 cycle a.c. train to the implanted area for .2 sec. The intensity of the current was adjusted to yield maximal bar pressing rates without siezure for each $\mathrm{S}$.

Phase II. Ten Ss, five hypothalamic and five septal implants, served in the discrimination phase, which also consisted of four daily sessions of $30 \mathrm{~min}$. each. The discrimination phase consisted of turning on a small $6.3 \mathrm{v}$ cue light above the bar when the bar was activated and reinforcement was available to S's electrode for each bar press. When the cue light was off no reinforcement was available to $s$. The cue light was actually on for a period of $15 \mathrm{~min}$. during the 30 min. session, but was randomly presented in intervals ranging for 15-60 sec. in length. Two Ss in the hypothalamic group and two Ss in the septal group served as controls and did not receive discrimination training so that the effects of discrimination training upon extinction performance could be estimated. Percent of responses made during light-on intervals was taken as the measure for discrimination.
Phase III. All 16 Ss were given a final $30 \mathrm{~min}$. session during which no bar presses were reinforced. The extinction measure was number of bar presses per animal during the session.

\section{Results and Discussion}

In Phase I, the acquisition phase, the hypothalamic implant group responded best, with a mean of 1548 responses per session for each animal. The septal
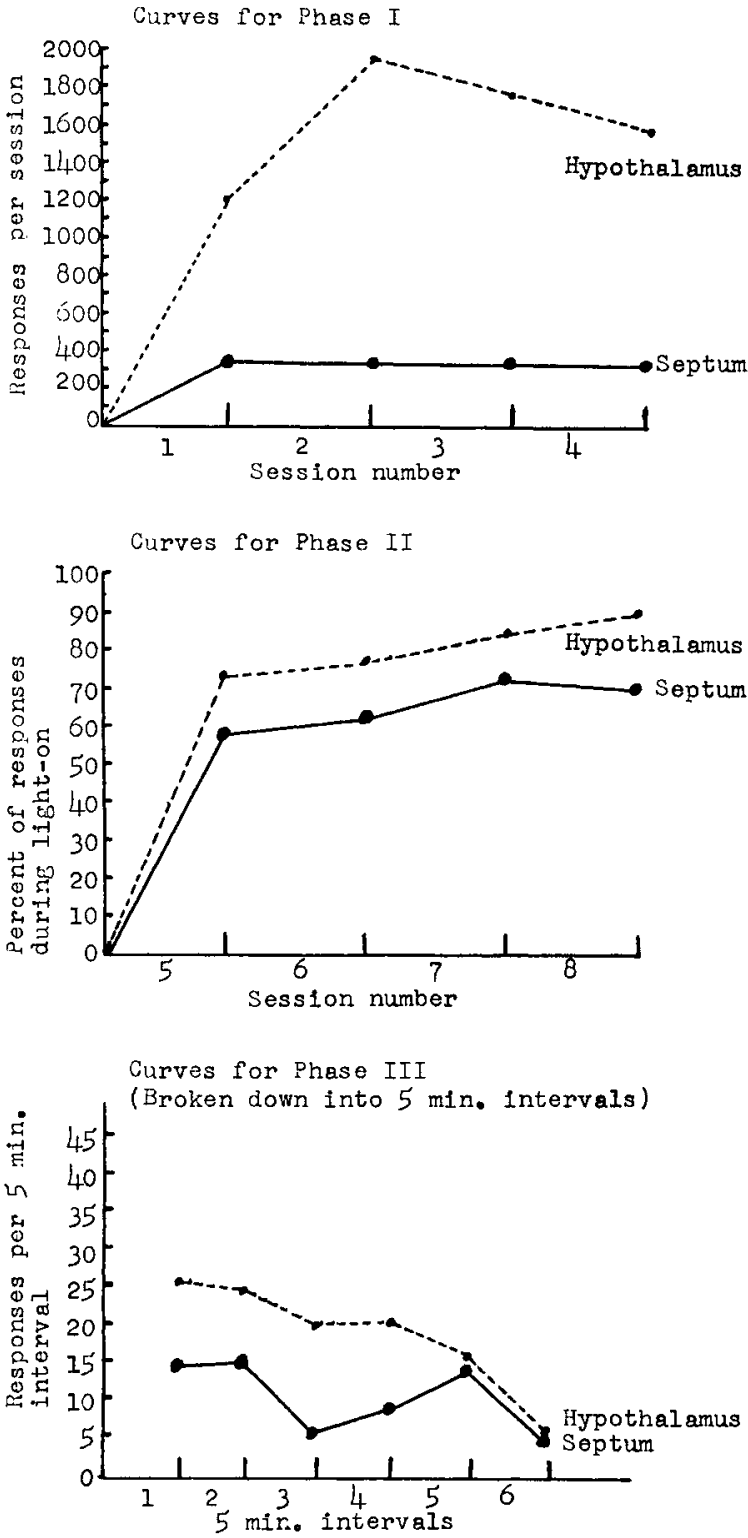
group was significantly lower with 337 responses per session. The nonimplanted control ss reflect the operant level of the apparatus with 63.4 responses per session. This data, then, supports the findings of other investigators that brain area is a major variable in rate of response for intracranial stimulation.

The data from Phase II also show a significant difference between brain areas. The hypothalamic implant group was again best with a mean of 79.8 percent of total responses made in the intervals during which the cue light was on, as compared to only 67.3 percent for the septal implant group. Data reported here indicate that locus of stimulation is an important factor in the equality of discrimination performance for intracranial stimulation.

Significant differences between brain areas are also shown in the results from Phase III. As in the other two phases, the hypothalamic implant group was significantly higher than the septal group. Ss in the hypothalamic group had a mean extinction score of 102.6 responses, while Ss in the septal group had a mean extinction score of 65.8. Nonimplanted control Ss had a mean of 40 responses during the final session.

It should be pointed out that discrimination training had no effect upon resistance to extinction. The two hypothalamic implants and the two septal implants which received training, but not discrimination training, did not differ from their group mates in extinction. This indicates that extinction scores were not inflated by the discrimination training, as might be expected in some cases.

It seems that the data obtained in Phase III, though

\section{Reply to Wechkin and Sackett}

by H. S. Pennypacker, James A. Horel, and Shirley A. Myers

In commenting on our previous paper (Myers, Horel, \& Pennypacker, 1965) Wechkin and Sackett raise two issues which evidently require clarification. The first, concerning the operant nature of the VR, is clearly definitional-they suggest that we have inadvertently identified as an operant VR a response which may in fact have been respondent. The second relates to the origin of vocal behavior and is at best, speculative and interpretational.

We have used the conventional definition of the word operant defined originally by Skinner (1938, p. 21) as "... an identifiable part of behavior of which it may be said not that no stimulus can be found that will elicit it (there may be a respondent, the response of which has the same topography) but that no correlated stimulus can be detected upon occasions when it is observed to occur." From the same reference we find respondent defined as "the kind of behavior correlated with specific eliciting stimuli..."

In our procedure one of a number of existing VRs is selectively reinforced and as typically happens in similar situations, the response increases in frequency based on only seven Ss in each of the two brain areas, have especially important implications because of the inconsistencies and contradictions found in previous studies investigating extinction with intracranial stimulation. Howarth \& Deutsch (1962) reported rapid extinction as a function of time, for instance, yet Herburg (1963) could not predict the effect of manipulating certain variables upon extinction with Deutsch's theory. These apparent inconsistencies could be easily reconciled by systematic demonstrations that the area stimulated is a major factor in the extinction curves for intracranial stimulation. The present investigation, though of only a preliminary nature, suggests that brain area is such a determiner.

Curves for all three phases are presented in the figure.

\section{References}

Herburg, L. J. Determinants of electrical self-stimulation. $J$. comp physiol. Psychol., 56, 686-690, 1963.

Howarth, C. I., \& Deutsch, J. A. Drive decay: The cause of fast extinction of habits learned for brain stimulation. Science, 137 . $35-36,1962$

olds, J. A preliminary mapping of electrical reinforcing effects in the rat brain. J. comp. phusiol. Psychol., 49, 3, 281-286, 1956.

Olds, J. Approach-avoidance dissociations in the rat brain. Amer. J. Physiol., 199, 965-968, 1960

Olds, M. F., \& Olds, J. Approach-avoidance analysis of rat diencephalon. J. comp. Neurol., 120, 2, 259-295, 1963.

\section{Notes}

1. Part of this research was reported at the 1965 meeting of the Rocky Mountain Psychological Association, Denver, Colorado.

2. Now at Kansas State University.

until a stable level of responding is achieved. Clearly, the food does not elicit the response, it follows it and "anticipation" of the food is clearly not an identifiable stimulus. Moreover, attention should be called to the fact that the vocal behavior in question was maintained on an FR 3 schedule; the classical conditioning literature is hardly replete with examples of responses maintained over long periods on a $33 \%$ reinforcement schedule (Kimble, 1964).

With regard to the origin of vocal behavior, our report simply did not address itself to that question. While it is true that modifying a response by operant methods in the laboratory does not prove that similar behavioral alterations in the natural setting are due to operant conditioning, neither does it preclude such a possibility. Nor does it preclude the unrelated possibility that the behavior initially functioned as a respondent; e.g., the human blink response appears to undergo such modification in the case of the unilateral lid closure which is a significant component of the courtship pattern.

In any case, even if the VR is a food call, we would ask what it serves the animal. Prior to conditioning, the response probably had some functional significance for the animal, but whether it was a food call or mating 